\title{
Generalizations of Hölder's and Some Related Integral Inequalities on Fractal Space
}

\author{
Guang-Sheng Chen \\ Department of Construction and Information Engineering, Guangxi Modern Vocational Technology College, Hechi, \\ Guangxi 547000, China
}

Correspondence should be addressed to Guang-Sheng Chen; cgswavelets@126.com

Received 5 May 2013; Accepted 8 July 2013

Academic Editor: Miguel Martin

Copyright (c) 2013 Guang-Sheng Chen. This is an open access article distributed under the Creative Commons Attribution License, which permits unrestricted use, distribution, and reproduction in any medium, provided the original work is properly cited.

Based on the local fractional calculus, we establish some new generalizations of Hölder's inequality. By using it, some related results on the generalized integral inequality in fractal space are investigated in detail.

\section{Introduction}

Let $p>1,1 / p+1 / q=1, f(x)$, and $g(x)$ be continuous real-valued functions on $[a, b]$. Then, the famous Hölders inequality reads as

$$
\int_{a}^{b}|f(x) g(x)| d x \leq\left(\int_{a}^{b}|f(x)|^{p} d x\right)^{1 / p}\left(\int_{a}^{b}|g(x)|^{q} d x\right)^{1 / q} .
$$

The renowned inequality of Hölder [1] is well celebrated for its beauty and its wide range of important applications to real and complex analysis and functional analysis, as well as many disciplines in applied mathematics. A large number of new proofs, various generalizations, refinements, variations, and applications of Hölder inequality have been investigated in the literature in [2-11]. Recently, it comes to our attention that an interesting local fractional integral Hölder's inequality, which was established by Yang [12], is as follows.

$$
\text { Let } f(x), g(x) \in C_{\alpha}(a, b), p>1,1 / p+1 / q=1 \text {. Then, }
$$

$$
\begin{aligned}
& \frac{1}{\Gamma(1+\alpha)} \int_{a}^{b}|f(x) g(x)|(d x)^{\alpha} \\
& \leq\left(\frac{1}{\Gamma(1+\alpha)} \int_{a}^{b}|f(x)|^{p}(d x)^{\alpha}\right)^{1 / p} \\
& \quad \times\left(\frac{1}{\Gamma(1+\alpha)} \int_{a}^{b}|g(x)|^{q}(d x)^{\alpha}\right)^{1 / q} .
\end{aligned}
$$

Recently, the local fractional calculus has attracted a lot of interest for scientists and engineers. Local fractional derivative had been introduced in [12-36]; that is the local fractional derivative was structured in [12-18, 26, 30-36], Jumarie gave the modified Riemann-Liouville derivative in $[19,20]$, the fractal derivative was considered in [21-25, 27-29], and the generalized fractal derivative was proposed by Chen et al. [25]. As a consequence, the theory of local fractional calculus becomes important for modelling problems for fractal mathematics and engineering on Cantor sets and it plays important role in many applications in several fields such as the theoretical physics $[14,18]$, the elasticity and fracture mechanics [14], the heat conduction theory [14, 16, 27], signal analysis [12, 13], the fluid mechanics [14], tensor analysis [14], Fourier and wavelet analysis $[12,13]$, optimization method [14], and complex analysis [12, 13]. For example, the local fractional Fokker-Planck equation was proposed in [18]. The local fractional Stieltjes transform was established in [37]. The fractal heat conduction problems were presented in $[14,27]$. Local fractional improper integral was obtained in [38]. The principles of virtual work, minimum potential, and complementary energy in the mechanics of fractal media were investigated in [14]. Mean value theorems for local fractional integrals were considered in [39]. The diffusion problems in fractal media were reported in [24].

The purpose of this work is to establish some generalizations of inequality (2) and give its corresponding reverse version. Moreover, the obtained results will be applied to 
establish local fractional integral reverse Minkowski inequality, Dresher's inequality, and its corresponding reverse version. This paper is divided into four sections. In Section 2, we recall some basic facts about local fractional calculus; in Section 3, we give some generalizations of the local fractional integral Hölder inequality and establish its corresponding reverse version; in Section 4, we apply the obtained results to establish reverse Minkowski inequality, Dresher's inequality, and its reverse form involving local fractional integral; some extensions of Minkowski and Dreshers inequalities are considered also.

\section{Preliminaries}

In this section, we recall the basic notions of local fractional calculus (see [12-14]).

2.1. Local Fractional Continuity of Functions. In order to study the local fractional continuity of nondifferentiable functions on fractal sets, we first give the following results.

Lemma 1 (see [14]). Assume that $F$ is a subset of the real line and is a fractal. Let $f:(F, d) \rightarrow\left(\Omega^{\prime}, d^{\prime}\right)$ be a bi-Lipschitz mapping. Then, there exist two positive constants $\rho, \tau$, and $F \subset$ $R$,

$$
\rho^{s} H^{s}(F) \leq H^{s}(f(F)) \leq \tau^{s} H^{s}(F)
$$

such that for all $x_{1}, x_{2} \in F$,

$$
\rho^{\alpha}\left|x_{1}-x_{2}\right|^{\alpha} \leq\left|f\left(x_{1}\right)-f\left(x_{2}\right)\right| \leq \tau^{\alpha}\left|x_{1}-x_{2}\right|^{\alpha} \text {. }
$$

From Lemma 1, we obtain easily

$$
\left|f\left(x_{1}\right)-f\left(x_{2}\right)\right| \leq \tau^{\alpha}\left|x_{1}-x_{2}\right|^{\alpha}
$$

such that

$$
\left|f\left(x_{1}\right)-f\left(x_{2}\right)\right| \leq \varepsilon^{\alpha},
$$

where $\alpha$ is fractal dimension of $F$. The result that is directly deduced from fractal geometry is related to fractal coarsegrained mass function $\gamma^{\alpha}[F, a, b]$ which reads

$$
\gamma^{\alpha}[F, a, b]=\frac{H^{\alpha}(F \cap(a, b))}{\Gamma(1+\alpha)}
$$

with

$$
H^{\alpha}(F \cap(a, b))=(b-a)^{\alpha},
$$

where $H^{\alpha}$ is $\alpha$ dimensional Hausdorff measure.

Notice that we consider the dimensions of any fractal spaces (e.g., Cantor spaces or like-Cantor spaces) as a positive number. It looks like Euclidean space because its dimension is also a positive number. The detailed results had been considered in [12-14].

Definition 2 (see $[12,14])$. If there exists

$$
\left|f(x)-f\left(x_{0}\right)\right| \leq \varepsilon^{\alpha},
$$

with $\left|x-x_{0}\right|^{\alpha} \leq \delta$, for $\varepsilon, \delta>0$ and $\varepsilon, \delta \in R$, then $f(x)$ is called local fractional continuous at $x=x_{0}$, denoted by $\lim _{x \rightarrow x_{0}} f(x)=f\left(x_{0}\right) . f(x)$ is local fractional continuous on the interval $(a, b)$, denoted by

$$
f(x) \in C_{\alpha}(a, b)
$$

if (9) holds for $x \in(a, b)$.

Definition 3 (see $[13,14]$ ). Assume that $f(x)$ is a nondifferentiable function of exponent $\alpha, 0<\alpha \leq 1$, which satisfies Hölder function of exponent $\alpha$, then, for $x, y \in X$ such that

$$
|f(x)-f(y)| \leq C|x-y|^{\alpha} \text {. }
$$

Definition 4 (see $[13,14]$ ). A function $f(x)$ is continuous of order $\alpha, 0<\alpha \leq 1$, or shortly $\alpha$ continuous, if

$$
\left|f(x)-f\left(x_{0}\right)\right| \leq o\left(\left(x-x_{0}\right)^{\alpha}\right) .
$$

Remark 5. Compared with (12), (9) is standard definition of local fractional continuity. Here, (11) is unified local fractional continuity [14].

\subsection{Local Fractional Derivatives and Integrals}

Definition 6 (see [12-14]). Let $f(x) \in C_{\alpha}(a, b)$. Local fractional derivative of $f(x)$ of order $\alpha$ at $x=x_{0}$ is given by

$$
f^{(\alpha)}\left(x_{0}\right)=\left.\frac{d^{\alpha} f(x)}{d x^{\alpha}}\right|_{x=x_{0}}=\lim _{x \rightarrow x_{0}} \frac{\Delta^{\alpha}\left(f(x)-f\left(x_{0}\right)\right)}{\left(x-x_{0}\right)^{\alpha}},
$$

where $\Delta^{\alpha}\left(f(x)-f\left(x_{0}\right)\right) \cong \Gamma(1+\alpha) \Delta\left(f(x)-f\left(x_{0}\right)\right)$.

For any $x \in(a, b)$, there exists

$$
f^{(\alpha)}(x)=D_{x}^{(\alpha)} f(x)
$$

denoted by

$$
f(x) \in D_{x}^{(\alpha)}(a, b) .
$$

Local fractional derivative of high order is derived as

$$
f^{(k \alpha)}(x)=\overbrace{D_{x}^{(\alpha)} \cdots D_{x}^{(\alpha)}}^{k \text { times }} f(x),
$$

and local fractional partial derivative of high order is derived as

$$
\frac{\partial^{k \alpha} f(x)}{\partial x^{k \alpha}}=\overbrace{\frac{\partial^{\alpha}}{\partial x^{\alpha}} \cdots \frac{\partial^{\alpha}}{\partial x^{\alpha}}}^{k \text { times }} f(x) .
$$

Definition 7 (see [12-14]). Let $f(x) \in C_{\alpha}(a, b)$. Local fractional integral of $f(x)$ of order $\alpha$ in the interval $[a, b]$ is deduced by

$$
\begin{aligned}
{ }_{a} I_{b}^{(\alpha)} f(x) & =\frac{1}{\Gamma(1+\alpha)} \int_{a}^{b} f(t)(d t)^{\alpha} \\
& =\frac{1}{\Gamma(1+\alpha)} \lim _{\Delta t \rightarrow 0} \sum_{j=0}^{N-1} f\left(t_{j}\right)\left(\Delta t_{j}\right)^{\alpha},
\end{aligned}
$$


where $\Delta t_{j}=t_{j+1}-t_{j}, \Delta t=\max \left\{\Delta t_{1}, \Delta t_{2}, \ldots, \Delta t_{j}, \ldots\right\}$, and $\left[t_{j}, t_{j+1}\right], j=1,2, \ldots, N-1, t_{0}=a, t_{N}=b$ is a partition of the interval $[a, b]$.

For convenience, we assume that

$$
\begin{aligned}
& { }_{\alpha} I_{\alpha}^{(\alpha)} f(x)=0 \quad \text { if } a=b, \\
& { }_{\alpha} I_{b}^{(\alpha)} f(x)=-{ }_{b} I_{\alpha}^{(\alpha)} f(x) \quad \text { if } a>b .
\end{aligned}
$$

For any $x \in(a, b)$, we can get

$$
{ }_{\alpha} I_{x}^{(\alpha)} f(x)
$$

denoted by

$$
f(x) \in{ }_{\alpha} I_{x}^{(\alpha)}(a, b) .
$$

Remark 8. If $f(x) \in D_{x}^{(\alpha)}(a, b)$, or ${ }_{\alpha} I_{x}^{(\alpha)}(a, b)$ then we have

$$
f(x) \in C_{\alpha}(a, b) \text {. }
$$

\section{Some Generalizations of Hölder Inequality and Its Reverse Form}

In the section, we give some generalizations of the inequality (2) and establish its reverse form.

Theorem 9 (reverse Hölder inequality). Let $f(x), g(x) \in$ $C_{\alpha}(a, b)$, and let $0<p<1,1 / p+1 / q=1$. Then,

$$
\begin{aligned}
& \frac{1}{\Gamma(1+\alpha)} \int_{a}^{b}|f(x) g(x)|(d x)^{\alpha} \\
& \geq\left(\frac{1}{\Gamma(1+\alpha)} \int_{a}^{b}|f(x)|^{p}(d x)^{\alpha}\right)^{1 / p} \\
& \quad \times\left(\frac{1}{\Gamma(1+\alpha)} \int_{a}^{b}|g(x)|^{q}(d x)^{\alpha}\right)^{1 / q} .
\end{aligned}
$$

Proof. Set $c=1 / p, q=-p d$, and then we have $d=c /(c-1)$. By inequality (2), we obtain

$$
\begin{aligned}
& \frac{1}{\Gamma(1+\alpha)} \int_{a}^{b}|f(x)|^{p}(d x)^{\alpha} \\
& =\frac{1}{\Gamma(1+\alpha)} \int_{a}^{b}|f(x) g(x)|^{p}|g(x)|^{-p}(d x)^{\alpha} \\
& \leq\left(\frac{1}{\Gamma(1+\alpha)} \int_{a}^{b}|f(x) g(x)|^{p c}(d x)^{\alpha}\right)^{1 / c} \\
& \quad \times\left(\frac{1}{\Gamma(1+\alpha)} \int_{a}^{b}|g(x)|^{-p d}(d x)^{\alpha}\right)^{1 / d} \\
& =\left(\frac{1}{\Gamma(1+\alpha)} \int_{a}^{b}|f(x) g(x)|(d x)^{\alpha}\right)^{1 / c} \\
& \quad \times\left(\frac{1}{\Gamma(1+\alpha)} \int_{a}^{b}|g(x)|^{q}(d x)^{\alpha}\right)^{1-p} .
\end{aligned}
$$

In (24), multiplying both sides by $((1 / \Gamma(1+$

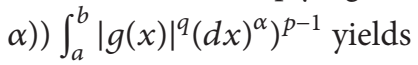

$$
\begin{aligned}
& \frac{1}{\Gamma(1+\alpha)} \int_{a}^{b}|f(x)|^{p}(d x)^{\alpha}\left(\frac{1}{\Gamma(1+\alpha)} \int_{a}^{b}|g(x)|^{q}(d x)^{\alpha}\right)^{p-1} \\
& \leq\left(\frac{1}{\Gamma(1+\alpha)} \int_{a}^{b}|f(x) g(x)|(d x)^{\alpha}\right)^{p} .
\end{aligned}
$$

Inequality (25) implies

$$
\begin{aligned}
& \frac{1}{\Gamma(1+\alpha)} \int_{a}^{b}|f(x) g(x)|(d x)^{\alpha} \\
& \geq\left(\frac{1}{\Gamma(1+\alpha)} \int_{a}^{b}|f(x)|^{p}(d x)^{\alpha}\right)^{1 / p} \\
& \quad \times\left(\frac{1}{\Gamma(1+\alpha)} \int_{a}^{b}|g(x)|^{q}(d x)^{\alpha}\right)^{1 / q} .
\end{aligned}
$$

Combining inequality (2) and Theorem 9, we can derive the following generalization.

Corollary 10. Let $f_{j}(x) \in C_{\alpha}(a, b)$, let $p_{j} \in R, j=1,2, \ldots m$, and let $\sum_{j=1}^{m} 1 / p_{j}=1$. Then,

(1) for $p_{j}>1$, one has

$$
\begin{aligned}
& \frac{1}{\Gamma(1+\alpha)} \int_{a}^{b} \prod_{j=1}^{m}\left|f_{j}(x)\right|(d x)^{\alpha} \\
& \leq \prod_{j=1}^{m}\left(\int_{a}^{b} \frac{1}{\Gamma(1+\alpha)}\left|f_{j}(x)\right|^{p_{j}}(d x)^{\alpha}\right)^{1 / p_{j}},
\end{aligned}
$$

(2) for $0<p_{1}<1, p_{j}<0, j=2, \ldots, m$, one has

$$
\begin{aligned}
& \frac{1}{\Gamma(1+\alpha)} \int_{a}^{b} \prod_{j=1}^{m}\left|f_{j}(x)\right|(d x)^{\alpha} \\
& \geq \prod_{j=1}^{m}\left(\int_{a}^{b} \frac{1}{\Gamma(1+\alpha)}\left|f_{j}(x)\right|^{p_{j}}(d x)^{\alpha}\right)^{1 / p_{j}} .
\end{aligned}
$$

Proof. (1) If $m=2, p_{1}, p_{2}$ are two positive constants and $1 / p_{1}+1 / p_{2}=1$. In particular, setting $p_{1}>1$, $p_{2}>1$, inequality (27) becomes inequality (2). Suppose (27) holds when $m \geq 2$. Using mathematical induction, let $p_{1}, p_{2}, \ldots, p_{m+1}>0$ be real numbers with $\sum_{j=1}^{m+1} 1 / p_{j}=1$ and $f_{j}(x) \geq 0, j=1,2, \ldots, m, m+1$; we must have $p_{i}>1$ for $j=1,2, \ldots, m, m+1$. In particular, we have

$$
p_{1}>0, \quad \frac{p_{1}}{p_{1}-1}>0, \quad \frac{1}{p_{1}}+\frac{p_{1}-1}{p_{1}}=1 .
$$


4

Journal of Function Spaces and Applications

By using Hölder's inequality (2), we obtain

$$
\begin{aligned}
& \frac{1}{\Gamma(1+\alpha)} \int_{a}^{b} \prod_{j=1}^{m+1}\left|f_{j}(x)\right|(d x)^{\alpha} \\
&=\frac{1}{\Gamma(1+\alpha)} \int_{a}^{b}\left|f_{1}(x)\right| \prod_{j=2}^{m+1}\left|f_{j}(x)\right|(d x)^{\alpha} \\
& \leq\left(\frac{1}{\Gamma(1+\alpha)} \int_{a}^{b}\left|f_{1}(x)\right|^{p_{1}}(d x)^{\alpha}\right)^{1 / p_{1}} \\
& \times\left(\frac{1}{\Gamma(1+\alpha)} \int_{a}^{b}\left(\prod_{j=2}^{m+1}\left|f_{j}(x)\right|\right)^{p_{1} /\left(p_{1}-1\right)}(d x)^{\alpha}\right)^{\left(p_{1}-1\right) / p_{1}} \\
&=\left(\frac{1}{\Gamma(1+\alpha)} \int_{a}^{b}\left|f_{1}(x)\right|^{p_{1}}(d x)^{\alpha}\right)^{1 / p_{1}} \\
& \times\left(\frac{1}{\Gamma(1+\alpha)} \int_{a}^{b} \prod_{j=2}^{m+1}\left|f_{j}(x)\right|^{p_{1} /\left(p_{1}-1\right)}(d x)^{\alpha}\right)^{\left(p_{1}-1\right) / p_{1}}
\end{aligned}
$$

since

$$
\begin{aligned}
& \frac{p_{j}\left(p_{1}-1\right)}{p_{1}}>0 \quad \text { for } j=2, \ldots, m, m+1, \\
& \begin{aligned}
\sum_{j=2}^{m+1} \frac{p_{1}}{p_{j}\left(p_{1}-1\right)} & =p_{1}\left(p_{1}-1\right) \sum_{j=2}^{m+1} \frac{1}{p_{j}} \\
& =p_{1}\left(p_{1}-1\right)\left(1-\frac{1}{p_{1}}\right)=1 .
\end{aligned}
\end{aligned}
$$

Using induction hypothesis and inequality (30), we can get

$$
\begin{aligned}
& \frac{1}{\Gamma(1+\alpha)} \int_{a}^{b} \prod_{j=1}^{m+1}\left|f_{j}(x)\right|(d x)^{\alpha} \\
& \leq\left(\frac{1}{\Gamma(1+\alpha)} \int_{a}^{b}\left|f_{1}(x)\right|^{p_{1}}(d x)^{\alpha}\right)^{1 / p_{1}} \\
& \times\left(\prod _ { j = 2 } ^ { m + 1 } \left(\frac{1}{\Gamma(1+\alpha)} \int_{a}^{b}\left|f_{j}(x)\right|^{p_{1} /\left(p_{1}-1\right) \cdot p_{j}\left(p_{1}-1\right) / p_{1}}\right.\right. \\
& \left.\left.=\left(\frac{1}{\Gamma(1+\alpha)} \int_{a}^{b}\left|f_{1}(x)\right|^{p_{1}}(d x)^{\alpha}\right)^{1 / p_{1}}\right)^{p_{1} / p_{j}\left(p_{1}-1\right)}\right)^{\left(p_{1}-1\right) / p_{1}} \\
& \times \prod_{j=2}^{m+1}\left(\frac{1}{\Gamma(1+\alpha)} \int_{a}^{b}\left|f_{j}(x)\right|^{p_{j}}(d x)^{\alpha}\right)^{1 / p_{j}} .
\end{aligned}
$$

Hence, this completes the proof.
(2) The Proof of (28) is similar to the proof of (27). Clearly when $m=2$, inequality (28) becomes Hölder's inequality (23). Now, suppose that (28) holds for some integer $m \geq 2$. Let $p_{1}, p_{2}, \ldots, p_{m}<0$, and let $p_{m+1} \in R$ be such that $\sum_{j=1}^{m+1} 1 / p_{j}=$ 1 , and let $f_{j}(x) \in L_{p, \alpha}[a, b], j=1,2, \ldots, m, m+1$. Note that $0<p_{m+1}<1$, since

$$
p_{1}>0, \quad 0<\frac{p_{1}}{p_{1}-1}<1, \quad \frac{1}{p_{1}}+\frac{p_{1}-1}{p_{1}}=1 .
$$

Observing Höder's inequality (23), we have

$$
\begin{aligned}
\frac{1}{\Gamma(1+\alpha)} \int_{a}^{b} \prod_{j=1}^{m}\left|f_{j}(x)\right|(d x)^{\alpha} \\
=\frac{1}{\Gamma(1+\alpha)} \int_{a}^{b}\left|f_{1}(x)\right| \prod_{j=2}^{m}\left|f_{j}(x)\right|(d x)^{\alpha} \\
\geq \\
\quad\left(\frac{1}{\Gamma(1+\alpha)} \int_{a}^{b}\left|f_{1}(x)\right|^{p_{1}}(d x)^{\alpha}\right)^{1 / p_{1}} \\
\quad \times\left(\frac{1}{\Gamma(1+\alpha)} \int_{a}^{b}\left(\prod_{j=2}^{m}\left|f_{j}(x)\right|\right)^{p_{1} /\left(p_{1}-1\right)}(d x)^{\alpha}\right)^{\left(p_{1}-1\right) / p_{1}} \\
\left.=\left(\frac{1}{\Gamma(\alpha)} \int_{a}^{b}\left|f_{1}(x)\right|^{p_{1}}(d x)^{\alpha}\right)^{1 / p_{1}} \int_{a}^{b} \prod_{j=2}^{m}\left|f_{j}(x)\right|^{p_{1} /\left(p_{1}-1\right)}(d x)^{\alpha}\right)^{\left(p_{1}-1\right) / p_{1}} \\
\quad \times\left(\frac{1}{\Gamma(1+\alpha)}\right.
\end{aligned}
$$

unless $(1 / \Gamma(1+\alpha)) \int_{a}^{b}\left|f_{1}(x)\right|^{p_{1}}(d x)^{\alpha}=0$.

Since

$$
\begin{gathered}
\frac{p_{j}\left(p_{1}-1\right)}{p_{1}}<0 \quad \text { for } j=2, \ldots, m, \\
\frac{p_{m+1}\left(p_{1}-1\right)}{p_{1}}>0, \\
\sum_{j=2}^{m+1} \frac{p_{1}}{p_{j}\left(p_{1}-1\right)}=1 .
\end{gathered}
$$

Combining induction hypothesis and (34), we obtain

$$
\begin{gathered}
\frac{1}{\Gamma(1+\alpha)} \int_{a}^{b} \prod_{j=1}^{m+1}\left|f_{j}(x)\right|(d x)^{\alpha} \\
\geq\left(\frac{1}{\Gamma(1+\alpha)} \int_{a}^{b}\left|f_{1}(x)\right|^{p_{1}}(d x)^{\alpha}\right)^{1 / p_{1}} \\
\times\left(\prod _ { j = 2 } ^ { m + 1 } \left(\frac{1}{\Gamma(1+\alpha)} \int_{a}^{b}\left|f_{j}(x)\right|^{p_{1} /\left(p_{1}-1\right) \cdot p_{j}\left(p_{1}-1\right) / p_{1}}\right.\right. \\
\left.\left.\times(d x)^{\alpha}\right)^{p_{1} / p_{j}\left(p_{1}-1\right)}\right)^{\left(p_{1}-1\right) / p_{1}}
\end{gathered}
$$


Journal of Function Spaces and Applications

5

$$
\begin{aligned}
& =\left(\frac{1}{\Gamma(1+\alpha)} \int_{a}^{b}\left|f_{1}(x)\right|^{p_{1}}(d x)^{\alpha}\right)^{1 / p_{1}} \\
& \quad \times \prod_{j=2}^{m+1}\left(\frac{1}{\Gamma(1+\alpha)} \int_{a}^{b}\left|f_{j}(x)\right|^{p_{j}}(d x)^{\alpha}\right)^{1 / p_{j}} \\
& =\prod_{j=1}^{m+1}\left(\frac{1}{\Gamma(1+\alpha)} \int_{a}^{b}\left|f_{j}(x)\right|^{p_{j}}(d x)^{\alpha}\right)^{1 / p_{j}}
\end{aligned}
$$

unless $(1 / \Gamma(1+\alpha)) \int_{a}^{b}\left|f_{j}(x)\right|^{p_{j}}(d x)^{\alpha}=0$ for some $j=$ $1,2, \ldots, m$.

4. Some Related Results

Theorem 11 (Minkowski inequality see [12]). Let $f(x)$, $g(x) \in C_{\alpha}(a, b), p>1$. Then,

$$
\begin{gathered}
\left(\frac{1}{\Gamma(1+\alpha)} \int_{a}^{b}|f(x)+g(x)|^{p}(d x)^{\alpha}\right)^{1 / p} \\
\leq\left(\frac{1}{\Gamma(1+\alpha)} \int_{a}^{b}|f(x)|^{p}(d x)^{\alpha}\right)^{1 / p} \\
\quad+\left(\frac{1}{\Gamma(1+\alpha)} \int_{a}^{b}|g(x)|^{q}(d x)^{\alpha}\right)^{1 / q} .
\end{gathered}
$$

Next, we give reverse version of inequality (37).

Theorem 12 (reverse Minkowski's inequality). Let $f(x)$, $g(x) \in C_{\alpha}(a, b), 0<p<1$. Then,

$$
\begin{gathered}
\left(\frac{1}{\Gamma(1+\alpha)} \int_{a}^{b}|f(x)+g(x)|^{p}(d x)^{\alpha}\right)^{1 / p} \\
\geq\left(\frac{1}{\Gamma(1+\alpha)} \int_{a}^{b}|f(x)|^{p}(d x)^{\alpha}\right)^{1 / p} \\
\quad+\left(\frac{1}{\Gamma(1+\alpha)} \int_{a}^{b}|g(x)|^{q}(d x)^{\alpha}\right)^{1 / q} .
\end{gathered}
$$

Proof. Let

$$
\begin{aligned}
M= & \frac{1}{\Gamma(1+\alpha)} \int_{a}^{b}|f(x)|^{p}(d x)^{\alpha}, \\
N= & \frac{1}{\Gamma(1+\alpha)} \int_{a}^{b}|g(x)|^{q}(d x)^{\alpha}, \\
W= & \left(\frac{1}{\Gamma(1+\alpha)} \int_{a}^{b}|f(x)|^{p}(d x)^{\alpha}\right)^{1 / p} \\
& +\left(\frac{1}{\Gamma(1+\alpha)} \int_{a}^{b}|g(x)|^{q}(d x)^{\alpha}\right)^{1 / q} .
\end{aligned}
$$


6

Journal of Function Spaces and Applications

$$
\begin{aligned}
= & \sum_{j=1}^{m}\left(\frac{1}{\Gamma(1+\alpha)} \int_{a}^{b}\left|f_{j}(x)\right|^{p}(d x)^{\alpha}\right)^{1 / p} \\
& \times\left(\frac{1}{\Gamma(1+\alpha)} \int_{a}^{b}\left|\sum_{j=1}^{m} f_{j}(x)\right|^{p}(d x)^{\alpha}\right)^{1 / q} .
\end{aligned}
$$

Multiplying $\left((1 / \Gamma(1+\alpha)) \int_{a}^{b}\left|\sum_{j=1}^{m} f_{j}(x)\right|^{p}(d x)^{\alpha}\right)^{1 / q}$ to two sides of (43), we get that (41) holds.

(2) The proof of (42) is similar to the proof of (38), so we omit it here.

Corollary 14. Let $f_{j}(x) \in C_{\alpha}(a, b), j=1,2, \ldots, m$. Then,

(1) for $p>1$, one has

$$
\begin{aligned}
& \frac{1}{\Gamma(1+\alpha)} \int_{a}^{b}\left|\sum_{j=1}^{m} f_{j}(x)\right|^{p}(d x)^{\alpha} \\
& >\sum_{j=1}^{m} \frac{1}{\Gamma(1+\alpha)} \int_{a}^{b}\left|f_{j}(x)\right|^{p}(d x)^{\alpha},
\end{aligned}
$$

(2) for $0<p<1$, one has

$$
\begin{aligned}
& \frac{1}{\Gamma(1+\alpha)} \int_{a}^{b}\left|\sum_{j=1}^{m} f_{j}(x)\right|^{p}(d x)^{\alpha} \\
& <\sum_{j=1}^{m} \frac{1}{\Gamma(1+\alpha)} \int_{a}^{b}\left|f_{j}(x)\right|^{p}(d x)^{\alpha} .
\end{aligned}
$$

Theorem 15 (Dresher's inequality). Let $f(x), g(x) \in C_{\alpha}(a, b)$, and let $0<r<1<p$; then

$$
\begin{gathered}
\left(\frac{(1 / \Gamma(1+\alpha)) \int_{a}^{b}|f(x)+g(x)|^{p}(d x)^{\alpha}}{(1 / \Gamma(1+\alpha)) \int_{a}^{b}|f(x)+g(x)|^{r}(d x)^{\alpha}}\right)^{1 /(p-r)} \\
\leq\left(\frac{(1 / \Gamma(1+\alpha)) \int_{a}^{b}|f(x)|^{p}(d x)^{\alpha}}{(1 / \Gamma(1+\alpha)) \int_{a}^{b}|f(x)|^{r}(d x)^{\alpha}}\right)^{1 /(p-r)} \\
+\left(\frac{(1 / \Gamma(1+\alpha)) \int_{a}^{b}|g(x)|^{p}(d x)^{\alpha}}{(1 / \Gamma(1+\alpha)) \int_{a}^{b}|g(x)|^{r}(d x)^{\alpha}}\right)^{1 /(p-r)} .
\end{gathered}
$$

Proof. Combining inequality (2) and Theorem 11, we have

$$
\begin{gathered}
\left(\frac{1}{\Gamma(1+\alpha)} \int_{a}^{b}|f(x)+g(x)|^{p}(d x)^{\alpha}\right)^{1 /(p-r)} \\
\leq\left(\left(\frac{1}{\Gamma(1+\alpha)} \int_{a}^{b}|f(x)|^{p}(d x)^{\alpha}\right)^{1 / p}\right. \\
\left.\quad+\left(\frac{1}{\Gamma(1+\alpha)} \int_{a}^{b}|g(x)|^{p}(d x)^{\alpha}\right)^{1 / p}\right)^{p /(p-r)}
\end{gathered}
$$

$$
\begin{gathered}
=\left(\left(\frac{\int_{a}^{b}|f(x)|^{p}(d x)^{\alpha}}{\int_{a}^{b}|f(x)|^{r}(d x)^{\alpha}}\right){ }^{1 / p}\right. \\
\times\left(\frac{1}{\Gamma(1+\alpha)} \int_{a}^{b}|f(x)|^{r}(d x)^{\alpha}\right)^{1 / p} \\
\left.\left.\left.+\left(\frac{\int_{a}^{b}|g(x)|^{p}(d x)^{\alpha}}{\int_{a}^{b}|g(x)|^{r}(d x)^{\alpha}}\right)^{1 / p}\right)^{b}|g(x)|^{r}(d x)^{\alpha}\right)^{1 / p}\right)^{p /(p-r)} \\
\times\left(\frac{1}{\Gamma(1+\alpha)} \int_{a}^{b}\right)^{1 /(p-r)} \\
\left.\leq\left(\frac{(1 / \Gamma(1+\alpha)) \int_{a}^{b}|f(x)|^{p}(d x)^{\alpha}}{(1 / \Gamma(1+\alpha)) \int_{a}^{b}|f(x)|^{r}(d x)^{\alpha}}\right)^{1 / r}|f(x)|^{r}(d x)^{\alpha}\right)^{r /(p-r)} \\
\times\left(\frac{1}{\Gamma(1+\alpha)} \int_{a}^{b}|g(x)|^{r}(d x)^{\alpha}\right)^{1 /(p-r)} \\
+\left(\frac{(1 / \Gamma(1+\alpha)) \int_{a}^{b}|g(x)|^{p}(d x)^{\alpha}}{(1 / \Gamma(1+\alpha)) \int_{a}^{b}|g(x)|^{r}(d x)^{\alpha}}\right)
\end{gathered}
$$

Using reverse Minkowski inequality (38), we have

$$
\begin{aligned}
& \left(\left(\frac{1}{\Gamma(1+\alpha)} \int_{a}^{b}|f(x)|^{r}(d x)^{\alpha}\right)^{1 / r}\right. \\
& \left.\quad+\left(\frac{1}{\Gamma(1+\alpha)} \int_{a}^{b}|g(x)|^{r}(d x)^{\alpha}\right)^{1 / r}\right)^{r} \\
& \leq \frac{1}{\Gamma(1+\alpha)} \int_{a}^{b}|f(x)+g(x)|^{r}(d x)^{\alpha} .
\end{aligned}
$$

By (47) and (48), we deduce that (46) holds. This completes the proof of the theorem.

Corollary 16. Let $f_{j}(x) \in C_{\alpha}(a, b), 0<r<1<p, j=$ $1,2, \ldots, m$. Then,

$$
\begin{gathered}
\left(\frac{(1 / \Gamma(1+\alpha)) \int_{a}^{b}\left|\sum_{j=1}^{m} f_{j}(x)\right|^{p}(d x)^{\alpha}}{(1 / \Gamma(1+\alpha)) \int_{a}^{b}\left|\sum_{j=1}^{m} f_{j}(x)\right|^{r}(d x)^{\alpha}}\right)^{1 /(p-r)} \\
\leq \sum_{j=1}^{m}\left(\frac{(1 / \Gamma(1+\alpha)) \int_{a}^{b}\left|f_{j}(x)\right|^{p}(d x)^{\alpha}}{(1 / \Gamma(1+\alpha)) \int_{a}^{b}\left|f_{j}(x)\right|^{r}(d x)^{\alpha}}\right)^{1 /(p-r)} .
\end{gathered}
$$


Theorem 17 (reverse Dresher's inequality). Let $f(x), g(x) \in$ $C_{\alpha}(a, b), p \leq 0 \leq r<1$. Then,

$$
\begin{gathered}
\left(\frac{(1 / \Gamma(1+\alpha)) \int_{a}^{b}|f(x)+g(x)|^{p}(d x)^{\alpha}}{(1 / \Gamma(1+\alpha)) \int_{a}^{b}|f(x)+g(x)|^{r}(d x)^{\alpha}}\right)^{1 /(p-r)} \\
\geq\left(\frac{(1 / \Gamma(1+\alpha)) \int_{a}^{b}|f(x)|^{p}(d x)^{\alpha}}{(1 / \Gamma(1+\alpha)) \int_{a}^{b}|f(x)|^{r}(d x)^{\alpha}}\right)^{1 /(p-r)} \\
+\left(\frac{(1 / \Gamma(1+\alpha)) \int_{a}^{b}|g(x)|^{p}(d x)^{\alpha}}{(1 / \Gamma(1+\alpha)) \int_{a}^{b}|g(x)|^{r}(d x)^{\alpha}}\right)^{1 /(p-r)} .
\end{gathered}
$$

Proof. Let $\alpha_{1} \geq 0, \alpha_{2} \geq 0, \beta_{1}>0, \beta_{2}>0$, and $-1<\lambda<0$, using Radon's inequality (see [3])

$$
\sum_{k=1}^{n} \frac{a_{k}^{p}}{b_{k}^{p-1}}<\frac{\left(\sum_{k=1}^{n} a_{k}\right)^{p}}{\left(\sum_{k=1}^{n} b_{k}\right)^{p-1}}, \quad a_{k} \geq 0, b_{k}>0,0<p<1 .
$$

We have

$$
\frac{\alpha_{1}^{\lambda+1}}{\beta_{1}^{\lambda}}+\frac{\alpha_{2}^{\lambda+1}}{\beta_{2}^{\lambda}} \leq \frac{\left(\alpha_{1}+\alpha_{2}\right)^{\lambda+1}}{\left(\beta_{1}+\beta_{2}\right)^{\lambda}},
$$

if and only if sequence $(\alpha)$ and sequence $(\beta)$ are proportional. Let

$$
\begin{aligned}
& \alpha_{1}=\left(\frac{1}{\Gamma(1+\alpha)} \int_{a}^{b}|f(x)|^{p}(d x)^{\alpha}\right)^{1 / p}, \\
& \beta_{1}=\left(\frac{1}{\Gamma(1+\alpha)} \int_{a}^{b}|f(x)|^{r}(d x)^{\alpha}\right)^{1 / r}, \\
& \alpha_{2}=\left(\frac{1}{\Gamma(1+\alpha)} \int_{a}^{b}|g(x)|^{p}(d x)^{\alpha}\right)^{1 / p}, \\
& \beta_{2}=\left(\frac{1}{\Gamma(1+\alpha)} \int_{a}^{b}|g(x)|^{r}(d x)^{\alpha}\right)^{1 / r},
\end{aligned}
$$

and set $\lambda=r /(p-r)$. Observing (52)-(53), we have

$$
\begin{aligned}
\frac{\alpha_{1}^{\lambda+1}}{\beta_{1}^{\lambda}}+\frac{\alpha_{2}^{\lambda+1}}{\beta_{2}^{\lambda}} & \left((1 / \Gamma(1+\alpha)) \int_{a}^{b}|f(x)|^{p}(d x)^{\alpha}\right)^{(\lambda+1) / p} \\
= & \frac{\left((1 / \Gamma(1+\alpha)) \int_{a}^{b}|f(x)|^{r}(d x)^{\alpha}\right)^{\lambda / r}}{\left((1 / \Gamma(1+\alpha)) \int_{a}^{b}|g(x)|^{r}(d x)^{\alpha}\right)^{\lambda / r}}
\end{aligned}
$$

$$
\begin{aligned}
& =\left(\frac{(1 / \Gamma(1+\alpha)) \int_{a}^{b}|f(x)|^{p}(d x)^{\alpha}}{(1 / \Gamma(1+\alpha)) \int_{a}^{b}|f(x)|^{r}(d x)^{\alpha}}\right)^{1 /(p-r)} \\
& +\left(\frac{(1 / \Gamma(1+\alpha)) \int_{a}^{b}|g(x)|^{p}(d x)^{\alpha}}{(1 / \Gamma(1+\alpha)) \int_{a}^{b}|g(x)|^{r}(d x)^{\alpha}}\right)^{1 /(p-r)} \\
& \leq \frac{\left(\alpha_{1}+\alpha_{2}\right)^{\lambda+1}}{\left(\beta_{1}+\beta_{2}\right)^{\lambda}} \\
& =\left(\left[\left(\frac{1}{\Gamma(1+\alpha)} \int_{a}^{b}|f(x)|^{p}(d x)^{\alpha}\right)^{1 / p}\right.\right.
\end{aligned}
$$$$
\left.\left.+\left(\frac{1}{\Gamma(1+\alpha)} \int_{a}^{b}|g(x)|^{p}(d x)^{\alpha}\right)^{1 / p}\right]^{p /(p-r)}\right)
$$$$
\times\left(\left[\left(\frac{1}{\Gamma(1+\alpha)} \int_{a}^{b}|f(x)|^{r}(d x)^{\alpha}\right)^{1 / r}\right.\right.
$$$$
\left.\left.+\left(\frac{1}{\Gamma(1+\alpha)} \int_{a}^{b}|g(x)|^{r}(d x)^{\alpha}\right)^{1 / r}\right]^{r /(p-r)}\right)^{-1} .
$$

Since $-1<\lambda=r /(p-r)<0$, let $p<0<r$, and let $0<r \leq 1$, and by Theorem 12, we obtain, respectively,

$$
\begin{aligned}
& {\left[\left(\frac{1}{\Gamma(1+\alpha)} \int_{a}^{b}|f(x)|^{p}(d x)^{\alpha}\right)^{1 / p}\right.} \\
& \left.+\left(\frac{1}{\Gamma(1+\alpha)} \int_{a}^{b}|g(x)|^{p}(d x)^{\alpha}\right)^{1 / p}\right]^{p} \\
& \geq \frac{1}{\Gamma(1+\alpha)} \int_{a}^{b}|f(x)+g(x)|^{p}(d x)^{\alpha},
\end{aligned}
$$

$$
\begin{aligned}
& {\left[\left(\frac{1}{\Gamma(1+\alpha)} \int_{a}^{b}|f(x)|^{r}(d x)^{\alpha}\right)^{1 / r}\right.} \\
& \left.+\left(\frac{1}{\Gamma(1+\alpha)} \int_{a}^{b}|g(x)|^{r}(d x)^{\alpha}\right)^{1 / r}\right]^{r} \\
& \quad \leq \frac{1}{\Gamma(1+\alpha)} \int_{a}^{b}|f(x)+g(x)|^{r}(d x)^{\alpha} .
\end{aligned}
$$

Observing (54)-(55), we obtain the desired results, and the theorem is completely proved. 
Corollary 18. Let $f_{j}(x) \in C_{\alpha}(a, b)$, and let $p \leq 0 \leq r<1$, $j=1,2, \ldots, m$. Then,

$$
\begin{gathered}
\left(\frac{(1 / \Gamma(1+\alpha)) \int_{a}^{b}\left|\sum_{j=1}^{m} f_{j}(x)\right|^{p}(d x)^{\alpha}}{(1 / \Gamma(1+\alpha)) \int_{a}^{b}\left|\sum_{j=1}^{m} f_{j}(x)\right|^{r}(d x)^{\alpha}}\right)^{1 /(p-r)} \\
\geq \sum_{j=1}^{m}\left(\frac{(1 / \Gamma(1+\alpha)) \int_{a}^{b}\left|f_{j}(x)\right|^{p}(d x)^{\alpha}}{(1 / \Gamma(1+\alpha)) \int_{a}^{b}\left|f_{j}(x)\right|^{r}(d x)^{\alpha}}\right)^{1 /(p-r)} .
\end{gathered}
$$

\section{Acknowledgments}

The authors would like to thank the anonymous referees for their valuable comments on the original version of this paper. This work was supported by the NNSFC (no. 11201433) and Scientific Research Project of Guangxi Education Department (no. 201204LX672).

\section{References}

[1] E. Hewitt and K. Stromberg, Real and Abstract Analysis. A Modern Treatment of the Theory of Functions of a Real Variable, Second Printing Corrected, Springer, New York, NY, USA, 1969.

[2] J. Kuang, Applied Inequalities, Shandong Science Press, Jinan, China, 2003.

[3] G. Hardy, J. E. Littlewood, and G. Pólya, Inequalities, Cambridge University Press, Cambridge, UK, 2nd edition, 1953.

[4] X. Yang, "A generalization of Hölder inequality," Journal of Mathematical Analysis and Applications, vol. 247, no. 1, pp. 328330, 2000.

[5] X. Yang, "Refinement of Hölder inequality and application to Ostrowski inequality," Applied Mathematics and Computation, vol. 138, no. 2-3, pp. 455-461, 2003.

[6] X. Yang, "A note on Hölder inequality" Applied Mathematics and Computation, vol. 134, no. 2-3, pp. 319-323, 2003.

[7] X. Yang, "Hölder's inequality," Applied Mathematics Letters, vol. 16, no. 6, pp. 897-903, 2003.

[8] S. Wu and L. Debnath, "Generalizations of Aczél's inequality and Popoviciu's inequality," Indian Journal of Pure and Applied Mathematics, vol. 36, no. 2, pp. 49-63, 2005.

[9] S. H. Wu, "Generalization of a sharp Hölder's inequality and its application," Journal of Mathematical Analysis and Applications, vol. 332, no. 1, pp. 741-750, 2007.

[10] S. Wu, "A new sharpened and generalized version of Hölder's inequality and its applications," Applied Mathematics and Computation, vol. 197, no. 2, pp. 708-714, 2008.

[11] E. G. Kwon and E. K. Bae, "On a continuous form of Hölder inequality," Journal of Mathematical Analysis and Applications, vol. 343, no. 1, pp. 585-593, 2008.

[12] X. Yang, Local Fractional Functional Analysis and Its Applications, Asian Academic publisher Limited, Hong Kong, 2011.

[13] X. J. Yang, "Local fractional integral transforms," Progress in Nonlinear Science, vol. 4, pp. 1-225, 2011.

[14] X. J. Yang, Advanced Local Fractional Calculus and Its Applications, World Science Publisher, New York, NY, USA, 2013.

[15] W.-H. Su, D. Baleanu, X.-J. Yang, and H. Jafari, "Damped wave equation and dissipative wave equation in fractal strings within the local fractional variational iteration method," Fixed Point Theory and Applications, vol. 2013, no. 1, pp. 89-102, 2013.
[16] M.-S. Hu, D. Baleanu, and X.-J. Yang, "One-phase problems for discontinuous heat transfer in fractal media," Mathematical Problems in Engineering, vol. 2013, Article ID 358473, 3 pages, 2013.

[17] K. M. Kolwankar and A. D. Gangal, "Hölder exponents of irregular signals and local fractional derivatives," Pramana, vol. 48, no. 1, pp. 49-68, 1997.

[18] K. M. Kolwankar and A. D. Gangal, "Local fractional FokkerPlanck equation," Physical Review Letters, vol. 80, no. 2, pp. 214217, 1998.

[19] G. Jumarie, "The Minkowski's space-time is consistent with differential geometry of fractional order," Physics Letters A, vol. 363, no. 1-2, pp. 5-11, 2007.

[20] G. Jumarie, "Modified Riemann-Liouville derivative and fractional Taylor series of nondifferentiable functions further results," Computers \& Mathematics with Applications, vol. 51, no. 9-10, pp. 1367-1376, 2006.

[21] A. Parvate and A. D. Gangal, "Calculus on fractal subsets of real line. I. Formulation," Fractals, vol. 17, no. 1, pp. 53-81, 2009.

[22] A. Parvate and A. D. Gangal, "Fractal differential equations and fractal-time dynamical systems," Pramana, vol. 64, no. 3, pp. 389-409, 2005.

[23] W. Chen, "Time-space fabric underlying anomalous diffusion," Chaos, Solitons and Fractals, vol. 28, no. 4, pp. 923-929, 2006.

[24] W. Chen, X. D. Zhang, and D. Korošak, "Investigation on fractional and fractal derivative relaxation-oscillation models," International Journal of Nonlinear Sciences and Numerical Simulation, vol. 11, no. 1, pp. 3-9, 2010.

[25] W. Chen, H. Sun, X. Zhang, and D. Korošak, "Anomalous diffusion modeling by fractal and fractional derivatives," Computers \& Mathematics with Applications, vol. 59, no. 5, pp. 1754-1758, 2010.

[26] F. B. Adda and J. Cresson, "About non-differentiable functions," Journal of Mathematical Analysis and Applications, vol. 263, no. 2, pp. 721-737, 2001.

[27] J. H. He, "A new fractal derivation," Thermal Science, vol. 15, no. 1, pp. 145-147, 2011.

[28] J.-H. He, "Asymptotic methods for solitary solutions and compactons," Abstract and Applied Analysis, vol. 2012, Article ID 916793, 130 pages, 2012.

[29] J. Fan and J. He, "Fractal derivative model for air permeability in hierarchic porous media," Abstract and Applied Analysis, vol. 2012, Article ID 354701, 7 pages, 2012.

[30] Y. J. Yang, D. Baleanu, and X. J. Yang, "A local fractional variational iteration method for Laplace equation within local fractional operators," Abstract and Applied Analysis, vol. 2013, Article ID 202650, 6 pages, 2013.

[31] F. Gao, X. Yang, and Z. Kang, "Local fractional newton's method derived from modified local fractional calculus," in Proceedings of the 2nd International Joint Conference on Computational Sciences and Optimization (CSO '09), pp. 228-232, IEEE Computer Society, April 2009.

[32] X. Yang and F. Gao, "The fundamentals of local fractional derivative of the one-variable nondifferentiable functions," Science \& Technology, vol. 31, no. 5, pp. 920-921, 2009.

[33] X. Yang and F. Gao, "Fundamentals of Local fractional iteration of the continuously non-differentiable functions derived from local fractional calculus," in Proceedings of the 2011 International Conference on Computer Science and Information Engineering (CSIE '11), pp. 398-404, Springer, 2011. 
[34] X. Yang, L. Li, and R. Yang, "Problems of local fractional definite integral of the one-variable nondifferentiable function," Science \& Technology, vol. 31, no. 4, pp. 722-724, 2009.

[35] X. Yang, L. Li, and R. Yang, "Problems of local fractional definite integral of the one-variable nondifferentiable function," Science \& Technology, vol. 31, no. 4, pp. 722-724, 2009.

[36] W.-H. Su, X.-J. Yang, H. Jafari, and D. Baleanu, "Fractional complex transform method for wave equations on Cantor sets within local fractional differential operator," Advances in Difference Equations, vol. 2013, no. 1, pp. 97-107, 2013.

[37] G. S. Chen, "The local fractional Stieltjes Transform in fractal space," Advances in Intelligent Transportation Systems, vol. 1, no. 1, pp. 29-31, 2012.

[38] G. S. Chen, "Local fractional Improper integral in fractal space," Advances in Information Technology and Management, vol. 1, no. 1, pp. 4-8, 2012.

[39] G. S. Chen, "Mean value theorems for local fractional integrals on fractal space," Advances in Mechanical Engineering and Its Applications, vol. 1, no. 1, pp. 5-8, 2012. 


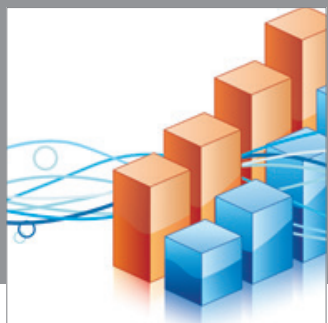

Advances in

Operations Research

mansans

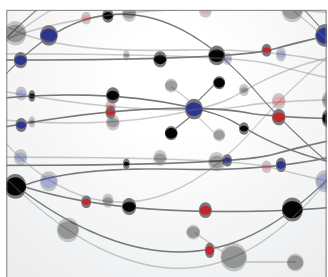

The Scientific World Journal
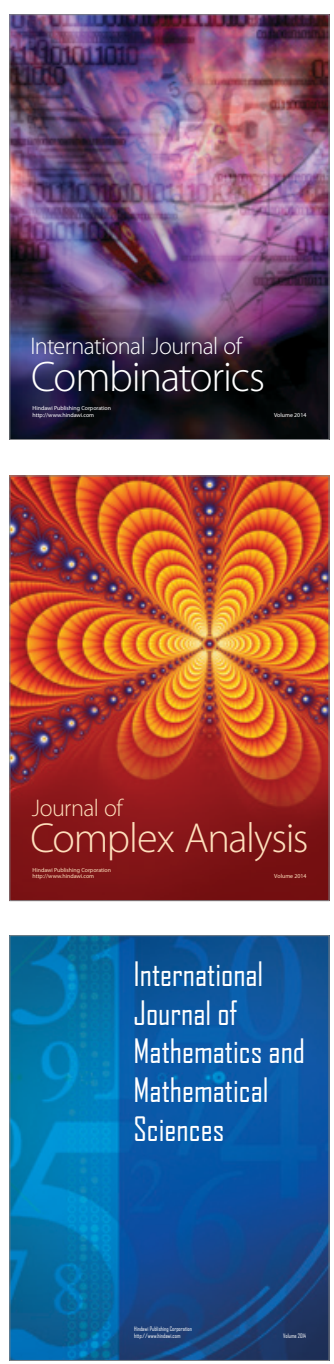
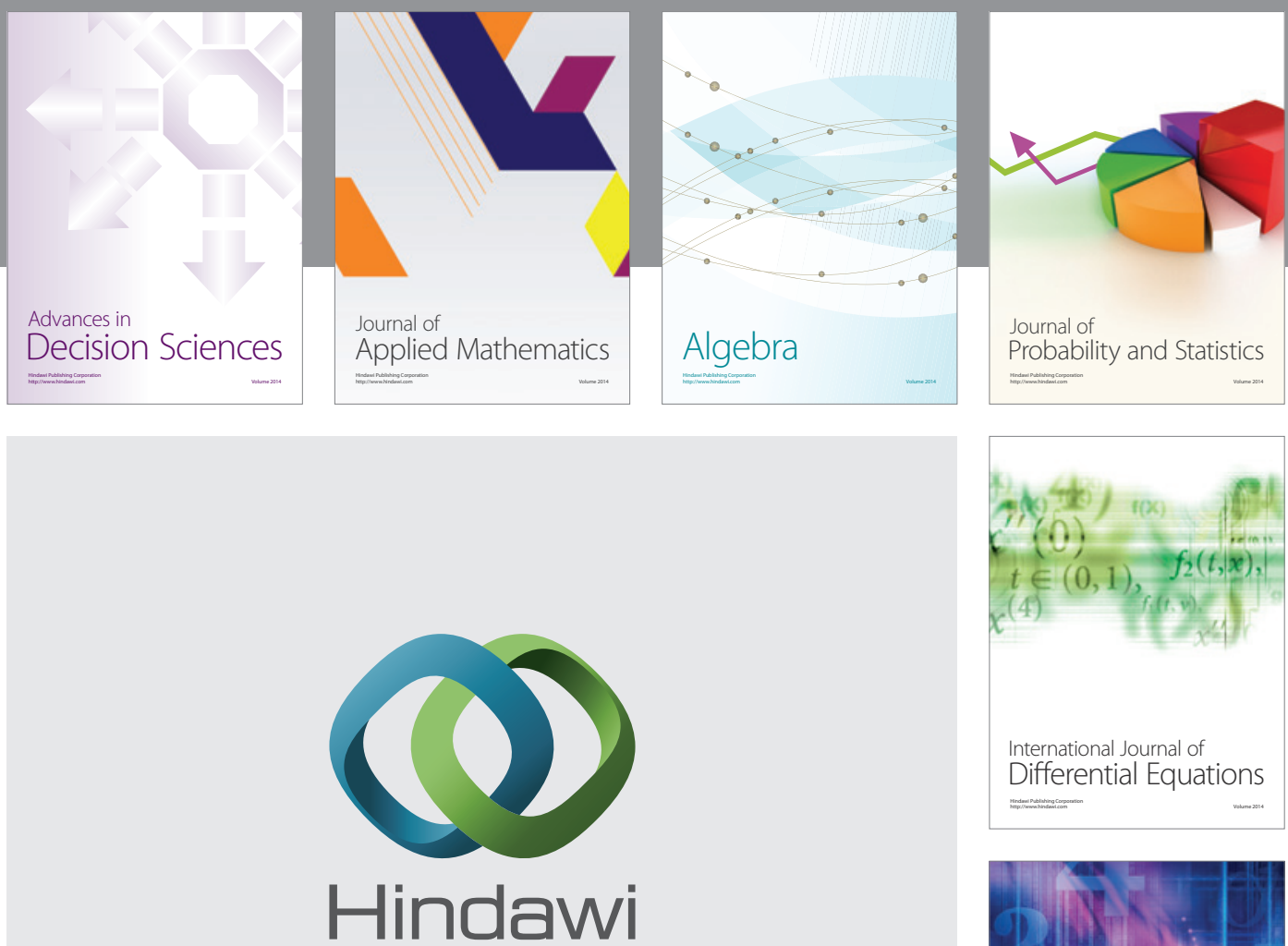

Submit your manuscripts at http://www.hindawi.com
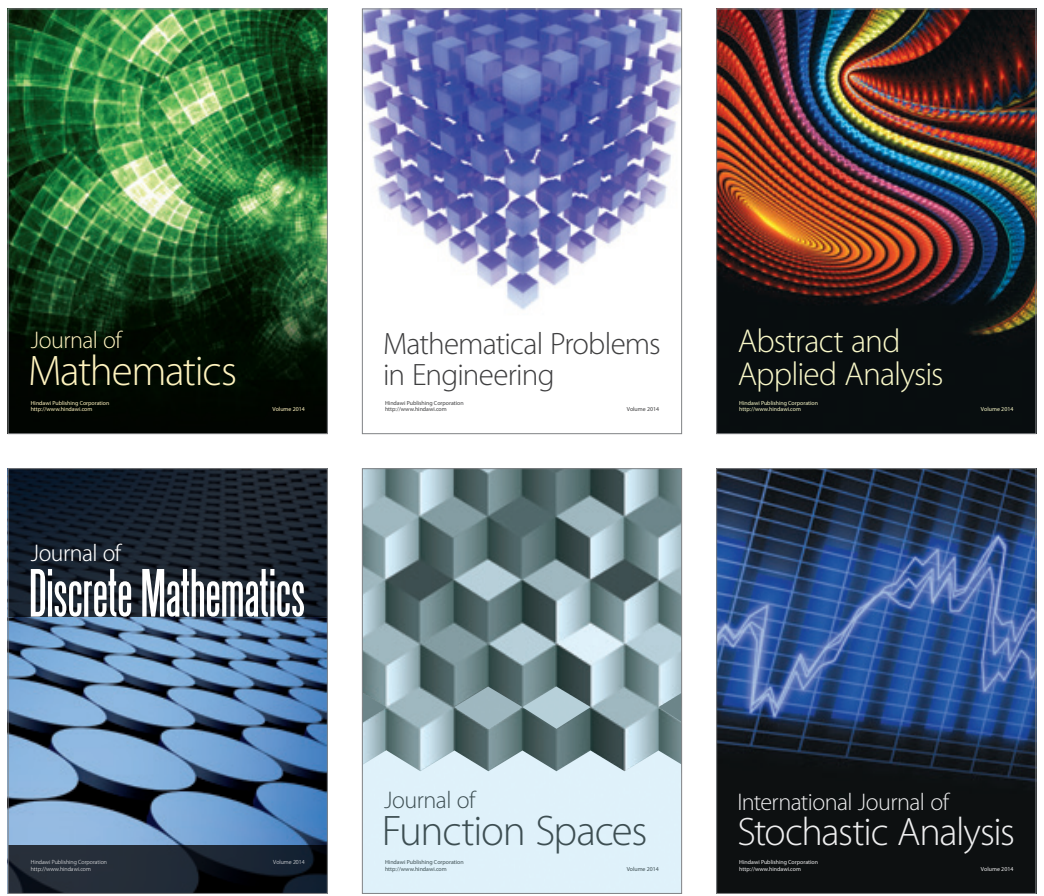

Journal of

Function Spaces

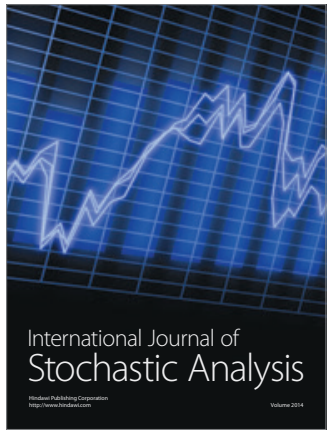

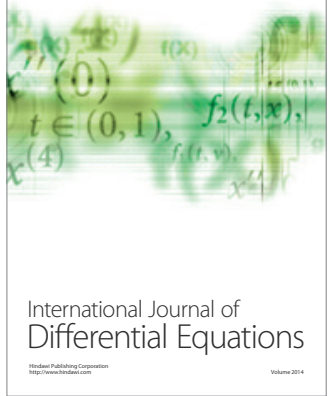
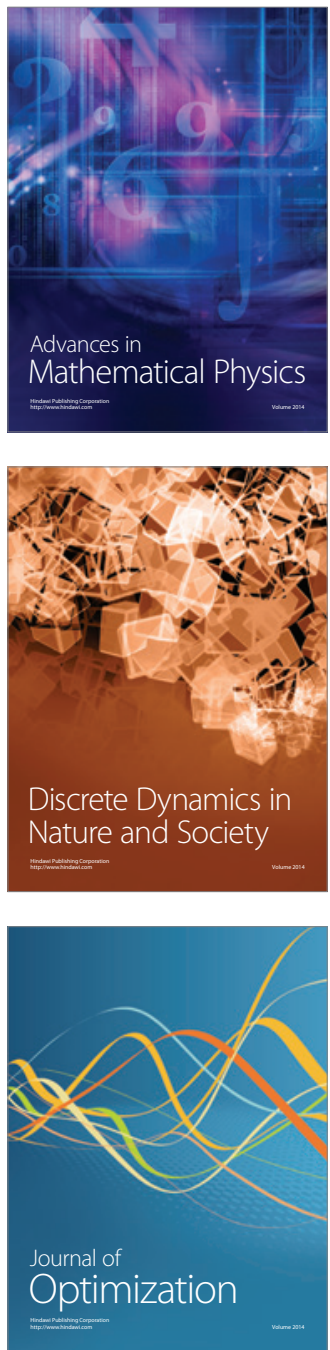